$$
R_{j k}=0,
$$$$
j+k<n-1 .
$$

Hence in the presence of (9.7) equations (9.5) and (9.6) are equivalent.

\title{
REFERENCES
}

1. W. F. Osgood, Lehrbuch der Funktionentheorie, vol. 2, part 1, Leipzig, 1929.

2. J. M. Thomas, Orderly differential systems, Duke Math. J. vol. 7 (1940) pp. 249-290.

DUKE UNIVERSITY

\section{RECENT INVESTIGATIONS OF THE SEPARATION OF LAPLACE'S EQUATION}

PARRY MOON AND DOMINA EBERLE SPENCER

Introduction. Two independent studies $[1 ; 2]$ have been made recently of separability conditions for the Laplace equation. Since the results of the two investigations are different, and even the meaning of "separability" is not identical, it seems pertinent to compare the two. We shall limit ourselves to euclidean 3-space and curvilinear coordinate systems $\left(u^{1}, u^{2}, u^{3}\right)$.

The meaning of "separability." According to [1], separation is of two kinds:

(a) If the assumption

$$
\phi=\prod_{i=1}^{3} U^{i}\left(u^{i}\right)
$$

permits the separation of the partial differential equation into three ordinary differential equations, the equation is said to be simply separable.

(b) If the assumption

$$
\phi=\frac{1}{R\left(u^{1}, u^{2}, u^{3}\right)} \prod_{i=1}^{3} U^{i}\left(u^{i}\right)
$$

permits the separation of the partial differential equation into three ordinary differential equations, and if $R \neq$ const., the equation is said to be $R$-separable.

These definitions seem to be in agreement with the usual meaning of the word "separability." Levinson, Bogert, and Redheffer [2],

Received by the editors June 11, 1952. 
however, choose a different meaning. This choice is, of course, perfectly legitimate; but unless the reader is aware of the difference, he may misinterpret the LBR theorems.

Instead of setting up the general criteria by which the Laplace equation in $n$ variables falls into $n$ separated equations, LBR split off one part at a time. Conditions are first determined by which terms in $u^{3}$ are separated; after which, additional restrictions are imposed so that $u^{1}$ can be separated from $u^{2}$.

It is customary to classify coordinate systems (with respect to solution of Laplace's equation) as separable systems and nonseparable systems. Redheffer [3], on the other hand, classifies coordinate systems as

(i) Those in which Laplace's equation can be split apart, one piece at a time. The equation is then said to be separable.

(ii) Those in which separated equations are obtainable but which do not allow a single solution to be split off. Redheffer calls these nonseparable but with separable solution.

(iii) All systems not in (i) and (ii). In the LBR paper [2], attention is confined to (i), while (ii) is ignored.

This tripart classification, though ingenious, does not appear to be very helpful. The practical question that always arises in the solution of Laplace's equation is "In the given coordinate system, can the partial differential equation be solved by the separation method, or must we resort to some other method?" The usual binary classification answers this question; the Redheffer classification does not.

Separation. The following theorems [1] apply:

THEOREM I. The necessary and sufficient condition for simple separability of the Laplace equation, in euclidean 3-space with an orthogonal coordinate system and $\phi=\phi\left(u^{1}, u^{2}, u^{8}\right)$, are

$$
\frac{g_{i i}}{g_{j j}}=\frac{M_{j 1}}{M_{i 1}}, \quad \frac{g^{1 / 2}}{g_{i i}}=f_{1}\left(u^{1}\right) f_{2}\left(u^{2}\right) f_{3}\left(u^{8}\right) M_{i 1},
$$

where $M_{i 1}$ is the cofactor of the element $\Phi_{i 1}$ in the Stäckel matrix,

$$
[S]=\left[\Phi_{i j}\left(u^{i}\right)\right], \quad i, j=1,2,3 .
$$

THEOREM II. The necessary and sufficient condition for $R$-separability of the Laplace equation, in euclidean 3-space with an orthogonal coordinate system and $\phi=\phi\left(u^{1}, u^{2}, u^{3}\right)$, is

$$
\sum_{i=1}^{3} \frac{1}{f_{i} g_{i i}} \frac{\partial}{\partial u^{i}}\left(f_{i} \frac{\partial R}{\partial u^{i}}\right)+\alpha_{1} \frac{R}{Q}=0,
$$


where $R$ and $Q$ are defined by the relations,

$$
g_{i i}=\frac{S}{M_{i 1}} Q, \quad \frac{g^{1 / 2}}{Q S}=f_{1}\left(u^{1}\right) f_{2}\left(u^{2}\right) f_{3}\left(u^{3}\right)(R)^{2} .
$$

The LBR results are most succinctly stated in the eight theorems of the abstract to the 1949 paper [2]. The first three theorems refer only to the splitting off of a single variable, and are therefore trivial. What we need is a criterion by which we can foretell, in the case of an unfamiliar coordinate system, whether three separation equations are obtainable. The Redheffer chipping process cannot be employed in finding this criterion because the requirements for the first step may be more restrictive than the general separability requirement.

A simple illustration brings out the difficulty. Consider Laplace's equation in paraboloidal coordinates $(\mu, \nu, \lambda)$ :

$$
\begin{aligned}
\nabla^{2} \phi= & \frac{((\mu-b)(\mu-c))^{1 / 2}}{(\mu-\nu)(\mu-\lambda)} \frac{\partial}{\partial \mu}\left(((\mu-b)(\mu-c))^{1 / 2} \frac{\partial \phi}{\partial \mu}\right) \\
& +\frac{((b-\nu)(c-\nu))^{1 / 2}}{(\mu-\nu)(\lambda-\nu)} \frac{\partial}{\partial \nu}\left(((b-\nu)(c-\nu))^{1 / 2} \frac{\partial \phi}{\partial \nu}\right) \\
& +\frac{((b-\lambda)(\lambda-c))^{1 / 2}}{(\mu-\lambda)(\lambda-\nu)} \frac{\partial}{\partial \lambda}\left(((b-\lambda)(\lambda-c))^{1 / 2} \frac{\partial \phi}{\partial \lambda}\right)=0 .
\end{aligned}
$$

To split off the $\lambda$-part (with $R=1$ ), we let

$$
\phi=S(\mu, \nu) \cdot Z(\lambda) \text {. }
$$

Substitution gives

$$
\begin{aligned}
(\lambda-\nu)[ & \left.\frac{((\mu-b)(\mu-c))^{1 / 2}}{S \cdot(\mu-\nu)} \frac{\partial}{\partial \mu}\left(((\mu-b)(\mu-c))^{1 / 2} \frac{\partial S}{\partial \mu}\right)\right] \\
& +(\mu-\lambda)\left[\frac{((b-\nu)(c-\nu))^{1 / 2}}{S \cdot(\mu-\nu)} \frac{\partial}{\partial \nu}\left(((b-\nu)(c-\nu))^{1 / 2} \frac{\partial S}{\partial \nu}\right)\right] \\
& +\left[\frac{((b-\lambda)(\lambda-c))^{1 / 2}}{Z} \frac{d}{d \lambda}\left(((b-\lambda)(\lambda-c))^{1 / 2} \frac{d Z}{d \lambda}\right)\right]=0
\end{aligned}
$$

which can be written in the form

$$
F_{1}(\lambda)+\lambda F_{2}(\mu, \nu)+F_{3}(\mu, \nu)=0 .
$$

Evidently there is no way of eliminating the $\lambda$ in the second term, so it is impossible to split off a differential equation in $\lambda$. The same difficulty arises with $\mu$ or with $\nu$. One concludes, therefore, that in 
paraboloidal coordinates Laplace's equation cannot be separated by Redheffer's method.

Yet everyone knows that it is separable. Applying the familiar equation,

$$
\frac{1}{f_{i}} \frac{d}{d u^{i}}\left(f_{i} \frac{d U^{i}}{d u^{i}}\right)+U^{i} \sum_{j=1}^{3} \alpha_{j} \Phi_{i j}=0,
$$

one obtains directly the three separated equations for paraboloidal coordinates:

$$
\begin{array}{r}
(\mu-b)(\mu-c) \frac{d^{2} \mathrm{M}}{d \mu^{2}}+\frac{1}{2}[2 \mu-(b+c)] \frac{d \mathrm{M}}{d \mu}-\left[\alpha_{2}-\alpha_{3} \mu\right] \mathrm{M}=0, \\
(6) \quad(b-\nu)(c-\nu) \frac{d^{2} \mathrm{~N}}{d \nu^{2}}+\frac{1}{2}[2 \nu-(b+c)] \frac{d \mathrm{~N}}{d \nu}-\left[\alpha_{2}-\alpha_{3} \nu\right] \mathrm{N}=0, \\
(b-\lambda)(\lambda-c) \frac{d^{2} \Lambda}{d \lambda^{2}}-\frac{1}{2}[2 \lambda-(b+c)] \frac{d \Lambda}{d \lambda}+\left[\alpha_{2}-\alpha_{3} \lambda\right] \Lambda=0 .
\end{array}
$$

Solution of these equations gives $M(\mu), N(\nu)$, and $\Lambda(\lambda)$; and particular solutions of Laplace's equation are

$$
\phi=\mathrm{M}(\mu) \cdot \mathrm{N}(\nu) \cdot \Lambda(\lambda) .
$$

Coordinate systems allowing separation. Let us compare the separable coordinate systems found by Redheffer with the systems which we know allow separation. LBR Theorem (IV) deals with coordinates that allow complete separation. According [2] to the statement on p. 261, Laplace's equation can be separated only in the following coordinate systems:

(a) Nine of the eleven Eisenhart systems [4],

(b) Toroidal coordinates,

(c) Inversions of (a) and (b).

The Redheffer method does not allow separation in general ellipsoidal or paraboloidal coordinates (Class IV of Eisenhart [4]), though Laplace's equation was actually separated in these coordinates by Lamé [5] before 1859 . LBR also discard many cyclide coordinate systems studied by Bôcher [6], Wangerin [7], and Darboux [8]. In particular, Bôcher [6] lists 17 classes of separable, cyclide coordinate systems. Theorem (IV) rejects many of these coordinates and their inversions. Theorems (V) and (VI) consider the special case of $R=1$, which has been correctly treated by Eisenhart [4] and others. LBR specifically exclude the general ellipsoidal and paraboloidal cases. 
Theorem (VII) states, "Coordinates for $R S Z$ or $R X Y Z$ separation of the Laplace equation have the group property under inversion." And on p. 261, "The set of coordinates giving separation is closed under inversion." The group property refers to a set of elements and a single operation, such that the set is closed with respect to this operation. In Redheffer's case, the set consists of all coordinate systems in which Laplace's equation separates. The operation is inversion in a sphere. Thus the theorem states that if we start with any system in which the Laplace equation is separable, we can obtain any other separable coordinate system by inversion. Starting with any coordinate system, by proper choice of centers of inversion, we can therefore obtain successively all the other elements of the group and finally arrive at the coordinate system with which we started.

The idea is, of course, untenable. As an example, take spherical coordinates. One family of coordinate surfaces are spheres, and it is well known that an inversion of a sphere is always a sphere. ${ }^{1} \mathrm{How}$, then, can any possible inversion or any succession of inversions transform the family of spheres into, say, a family of paraboloids?

Regarding the totality of coordinate systems which allow separation, Redheffer [9] lists the "complete" tabulation of separable systems, "giving the linear element $(d s)^{2}$ for all euclidean coordinate systems in which Laplace's equation separates." Among the 13 tabulated classes, one is surprised to find that 4 refer to the same coordinate system (conical coordinates). The remaining items do not include general ellipsoidal or paraboloidal coordinates or any of the more general cyclide coordinates or their inversions.

One may wonder if there is any advantage in trying to list all possible coordinates in which the Laplace equation separates. Including asymmetric inversions, there appear to be at least 60 such distinct systems, many of which are so mathematically complex that they will never be used. On the whole, therefore, it may be wiser to concentrate on the coordinate systems [10] which are most promising from a practical standpoint.

\section{REFERENCES}

1. P. Moon and D. E. Spencer, Theorems on separability in Riemannian n-space, Proc. Amer. Math. Soc. vol. 3 (1952) p. 635.

2. N. Levinson, B. Bogert, and R. M. Redheffer, Separation of Laplace's equation, Quarterly of Applied Mathematics vol. 7 (1949) p. 241.

3. R. M. Redheffer, Separation of Laplace's equation (Massachusetts Institute of Technology Ph.D. Thesis, 1948); Research Laboratory of Electronics, M.I.T., Technical Report no. 68, June 2, 1948.

\footnotetext{
${ }^{1}$ Including the plane as a special case.
} 
4. L. P. Eisenhart, Separable systems of Stäckel, Ann. of Math. vol. 35 (1934) p. 284.

5. G. Lamé, Legons sur les coordonnees curvilignes et leurs diverses applications, Paris, 1859.

6. M. Bocher, Über die Reihenentwickelungen der Potentialtheorie, Leipzig, Teubner, 1894. (See particularly the list of separable cyclide systems on p. 102 and the pictures on pp. 72 to 85); C. Segre, Etude des différentes surfaces $d u 4^{\circ}$ ordre d conique double ou cuspidale, Math. Ann. vol. 24 (1884) p. 313.

7. A. Wangerin, Reduction der Potentialgleichung für gewisse Rotationskörper auf eine gewöhnliche Differentialgleichung, Preis. d. Jablonowski Ges., Leipzig, no. 18, 1875; Über ein dreifach orthogonales Flächensystem, gebildet aus gewissen Flächen vierter Ordnung, J. Reine Angew. Math. vol. 82 (1877) pp. 145, 348; Über die Reduction der Gleichung (Laplace) auf gewöhnliche Differentialgleichungen, Monatsber. Kön. Akad. d. Wissen. Berlin, 1878, p. 152.

8. G. Darboux, Sur l'application des methodes de la physique mathematique d' l'etude des corps termines par des cyclides, C. R. Acad. Sci. Paris vol. 83 (1876) pp. 1037, 1099; vol. 84 (1877) p. 298.

9. R. M. Redheffer, Tech. Report No. 68 , p. 60.

10. P. Moon and D. E. Spencer, Separability in a class of coordinate systems, J. Franklin Inst. vol. 254 (1952) p. 227; Cylindrical and rotational coordinate systems, J. Franklin Inst. vol. 252 (1951) p. 327; Coordinate systems derived from circles, to be published.

Massachusetts Institute of Technology and UnIVERSITY OF CoNNecticut 